University of Puget Sound

Sound Ideas

All Faculty Scholarship

Faculty Scholarship

$1-1-1984$

\title{
Fonction et origine probable du point équant de Ptolémée
}

James C. Evans

University of Puget Sound, jcevans@pugetsound.edu

Follow this and additional works at: http://soundideas.pugetsound.edu/faculty_pubs

\section{Citation}

Evans, James C. 1984. "Fonction et origine probable du point équant de Ptolémée." Revue D'histoire Des Sciences 37(3-4): 193-213.

This Article is brought to you for free and open access by the Faculty Scholarship at Sound Ideas. It has been accepted for inclusion in All Faculty Scholarship by an authorized administrator of Sound Ideas. For more information, please contact soundideas@pugetsound.edu. 


\section{Fonction et origine probable du point équant de Ptolémée.}

In: Revue d'histoire des sciences. 1984, Tome 37 n³-4. pp. 193-213.

\section{Résumé}

RÉSUMÉ. - En partant toujours des apparences, nous examinons les fonctions du point équant et du cercle déférent excentrique dans la théorie planétaire de Ptolémée. Nous démontrons que la nécessité du point équant devient évidente aussitôt que l'on essaye de sauver les longueurs des arcs rétrogrades, et les intervalles entre eux, particulièrement dans le cas de Mars. Enfin, nous proposons une hypothèse sur la démarche qui a conduit Ptolémée à la découverte du point équant.

\section{Abstract}

SUMMARY. - Beginning with the essential planetary phenomena to be explained, we examine the functions of the equant point and the eccentric deferent circle in Ptolemy's theory. We show that the necessity of the equant point becomes evident as soon as one attempts to save the lengths of the retrograde arcs, and the distances between them, particularly in the case of Mars. Finally, we propose a hypothesis concerning the line of investigation that led Ptolemy to discover the equant.

Citer ce document / Cite this document :

EVANS JAMES. Fonction et origine probable du point équant de Ptolémée. In: Revue d'histoire des sciences. 1984 , Tome 37 n³-4. pp. 193-213.

doi : $10.3406 /$ rhs. 1984.2036

http://www.persee.fr/web/revues/home/prescript/article/rhs_0151-4105_1984_num_37_3_2036 


\section{Fonction et origine probable du point équant de Ptolémée}

RESUME. - En partant toujours des apparences, nous examinons les fonc. tions du point équant et du cercle déférent excentrique dans la théorie planétaire de Ptolémée. Nous démontrons que la nécessité du point équant devient évidente aussitôt que l'on essaye de sauver les longueurs des arcs rétrogrades, et les intervalles entre eux, particulièrement dans le cas de Mars. Enfin, nous proposons une hypothèse sur la démarche qui a conduit Ptolémée à la découverte du point équant.

SUMMARY. - Beginning with the essential planetary phenomena to be explained, we examine the functions of the equant point and the eccentric deferent circle in Ptolemy's theory. We show that the necessity of the equant point becomes evident as soon as one attempts to save the lengths of the retrograde arcs, and the distances between them, particularly in the case of Mars. Finally, we propose a hypothesis concerning the line of investigation that led Ptolemy to discover the equant.

Tout le monde connaît, fait assez remarquable, les traits les plus importants du système planétaire de Ptolémée $\left({ }^{*}\right)$. La figure 1 montre la théorie des longitudes retenue par Ptolémée pour Vénus, Mars, Jupiter et Saturne (la théorie de Mercure comporte une complication supplémentaire). Le plan de la figure est le plan de l'écliptique. Le point $C$ est le centre du cercle déférent AKII. La Terre se trouve au point $O$. Le cercle déférent, donc, n'est pas concentrique à la Terre, mais excentrique. La ligne qui passe par les points $O$ et $C$ coupe le déférent à l'apogée $A$, et au périgée $I I$ du cercle. Cette ligne, sur laquelle se trouvent les points $A, C, O$ et $I$, s'appelle la ligne des apsides. Le centre $\mathrm{K}$ de l'épicycle se déplace sur le déférent. Son

(*) Je voudrais remercier mes amis les Tellier et les membres de l'Equipe d'histoire de l'astronomie de l'Observatoire de Paris, qui m'ont apporté une grande aide dans la rédaction de cet article.

Rev. Hist. Sci., 1984, XXXVII/3-4

RHS - 7 


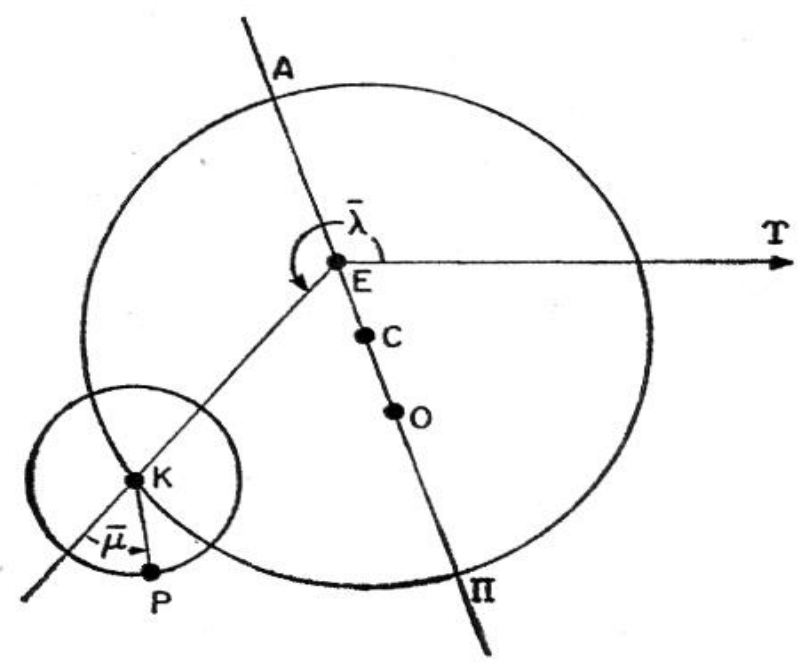

FIg. 1. - Théorie des longitudes de Ptolémée pour Vénus, Mars, Jupiter et Saturne

mouvement n'est uniforme, ni vu de la Terre $O$, ni vu du centre $C$ du cercle déférent; en revanche il est uniforme vu d'un troisième point $E$, centre du mouvement uniforme, ou point équant : cela veut dire qu'un observateur imaginaire placé au point $\mathbf{E}$ verrait se déplacer le point $K$ à une vitesse angulaire constante. Pour des observateurs placés en $O$ ou en $C$, le mouvement n'est pas uniforme. Pour un observateur sur la Terre, le point $K$ se déplace le plus vite quand il est près du périgée, et le plus lentement quand il est près de l'apogée.

Les ouvrages de vulgarisation consacrés à l'histoire de l'astronomie ont été souvent sévères pour Ptolémée et son système. Dans un style mélodramatique, on traite Ptolémée et Aristote comme les scélérats d'une histoire dont les héros sont Copernic, Tycho Brahé et Kepler. On pourrait citer une dizaine d'exemples, mais il suffira d'un seul. Après avoir décrit le système de Ptolémée - la combinaison d'un cercle déférent et d'un épicycle, et les trois centres (Terre, centre du déférent et point équant) - , Rudolph Thiel avoue que le système réussit comme modèle mathématique. Puis il commence à récriminer :

" Mais qu'en était-il de la signification du Tout, qu'en était-il pour la cosmologie grecque ? Les planètes se déplaçaient maintenant en boucles, c'est-à-dire autour d'un point imaginaire qui, lui, pour une raison inconnue, tournait autour de la Terre. Cependant, même ce point imaginaire ne tournait pas exactement autour de la Terre; le centre de son cercle était un deuxième point imaginaire à côté de la Terre; et, puisque cela ne donnait pas encore un mouvement uniforme, un troisième point imaginaire était nécessaire, autour duquel le mouvement paraissait être uniforme. 
«Voilà le dernier tableau de Ptolémée ; voilà le dernier mot de l'astronomie grecque ! Quelle absurdité ! Quelle capitulation pour l'esprit humain d'accepter un tel mécanisme de l'univers. Quelle triste fin pour l'harmonie des sphères " (1)!

Cette attitude, sous des formes plus modérées, est assez répandue. Elle provient, en partie, de la difficulté à comprendre la fonction du déférent excentrique et surtout celle du point équant. Mais, en vérité, ces détails du système de Ptolémée proviennent très naturellement de l'observation des rétrogradations des planètes, principalement des planètes supérieures.

Le but de cet article est d'abord de démontrer, aussi simplement que possible, la fonction de chaque détail du système destiné à sauver les phénomènes, et ensuite de proposer une hypothèse sur la démarche qui a conduit Ptolémée à la découverte du point équant.

\section{INTRODUCTION AUX PHÉNOMĖNES}

On appelle inégalité ou anomalie du mouvement d'un corps céleste tout écart à une vitesse uniforme et constante. Cette terminologie est liée à la méthode de calcul chez les astronomes, anciens aussi bien que modernes. On calcule d'abord la position moyenne du corps, c'est-à-dire la position qu'il occuperait s'il n'y avait pas d'inégalité. Ensuite, on ajoute ou soustrait une petite correction, ou « équation ", qui mesure l'écart entre la position moyenne et la position vraie.

\section{Inégalité zodiacale du Soleil}

Chaque planète a deux inégalités distinctes qui, dans le ciel, sont combinées. Le Soleil, cependant, n'a qu'une seule inégalité. Donc un examen bref de la théorie grecque du Soleil servira de bonne introduction à la théorie des planètes, dont les mouvements sont plus compliqués.

La seule inégalité dans le mouvement du Soleil s'appelle quelquefois inégalité (ou anomalie) zodiacale, parce que le Soleil paraît se déplacer moins vite dans certains signes du zodiaque que dans

(1) Rudolph Thiel, And There Was Light, trad. angl. par Richard et Clara Winston, New York, Mentor Books, 1960, p. 60. Publié en allemand sous le titre Und es ward Licht (Hamburg, Rowohlt Verlag, 1956). 
d'autres. A l'époque actuelle, le mouvement du Soleil est le moins rapide au mois de juillet, lorsque le Soleil est dans le Cancer.

Cette inégalité de vitesse est rendue sensible par l'inégalité de longueur des saisons. A notre époque, les équinoxes et les solstices ont lieu le plus souvent aux dates suivantes :

$\begin{array}{ll}\text { Equinoxe de printemps } & 21 \text { mars } \\ \text { Solstice d'été } & 22 \text { juin } \\ \text { Equinoxe d'automne } & 23 \text { septembre } \\ \text { Solstice d'hiver } & 22 \text { décembre }\end{array}$

On trouve les longueurs des saisons simplement en comptant les jours sur un calendrier :

$\begin{array}{ll}\text { Printemps } & 93 \text { jours, à peu près } \\ \text { Eté } & 93 \text { jours } \\ \text { Automne } & 90 \text { jours } \\ \text { Hiver } & 89 \text { jours }\end{array}$

Il y a donc 7 jours de plus de l'équinoxe de printemps à l'équinoxe d'automne que de l'équinoxe d'automne à l'équinoxe de printemps suivant.

Il semble que ce soit au ve siècle avant J.-C. que les astronomes grecs ont remarqué des différences entre les saisons, mais les valeurs des longueurs des saisons finalement retenues ont été celles d'Hipparque ( $I^{e}$ siècle av. J.-C.)

\begin{tabular}{|c|c|}
\hline Printemps & $941 / 2$ jours \\
\hline Eté & $921 / 2$ jours \\
\hline Automne & $88 \quad 1 / 8$ \\
\hline Hiver & $901 / 8$ \\
\hline
\end{tabular}

On remarque que ces valeurs ne sont pas les mêmes que celles d'aujourd'hui. Mais ce n'est pas une erreur d'Hipparque - les longueurs des saisons ont vraiment changé. Autrefois, c'était l'automne qui était la saison la plus courte : aujourd'hui, c'est l'hiver.

Donc le Soleil ne se déplace pas avec une vitesse angulaire uniforme. Aujourd'hui, il lui faut 93 jours pour parcourir les $90^{\circ}$ qui séparent le solstice d'été de l'équinoxe d'automne, mais seulement 89 jours pour les $90^{\circ}$ qui séparent le solstice d'hiver de l'équinoxe de printemps. Et donc le soleil se déplace un peu plus vite en janvier qu'en juillet. Cette variation apparente s'appelle anomalie ou inégalité solaire. C'est un exemple d'inégalité zodiacale, parce que la vitesse du Soleil est seulement une fonction de sa position dans le zodiaque. 
Afin d'expliquer l'inégalité solaire, il fallait que les astronomes grecs abandonnassent une de leurs trois hypothèses de départ : 1) l'orbite du Soleil est un cercle ; 2) le centre du cercle est la Terre ; 3) le Soleil se déplace le long du cercle à vitesse constante. On sait aujourd'hui que les trois hypothèses sont fausses. En effet, l'orbite est une ellipse; la Terre ne se trouve pas au centre de l'ellipse, mais à l'un de ses foyers; et la vitesse du Soleil n'est pas constante. Cependant au $\mathrm{II}^{\mathrm{e}}$ siècle av. J.-C., il aurait été déraisonnable de rejeter une théorie qui n'exigeait qu'une petite modification pour s'adapter aux apparences. Il aurait été douloureux d'abandonner ou l'orbite circulaire ou la vitesse constante : cela aurait détruit la simplicité du système et compliqué le calcul.

Ce fut Hipparque, ou peut-être Apollonius, qui démontra que l'on pouvait sauver les phénomènes par une modification bien moins grave. Le Soleil se déplace encore le long d'un cercle à une vitesse constante, mais le centre du cercle n'est plus la Terre : il en est un peu écarté. La figure 2 montre la position du centre du cercle par rapport à la Terre qui, de nos jours, dans ce système, rendrait compte des apparences. Le point $C$ est le centre du cercle excentrique du Soleil. Le point $O$ est le centre du monde, c'est-à-dire la Terre. Pour un observateur terrestre, la distance angulaire entre un point équinoxial et un point solsticial est toujours de $90^{\circ}$, mais ces points ne divisent plus le cercle du Soleil en quatre intervalles égaux. Nous obtenons donc des saisons de longueurs inégales : l'été est la saison la plus longue, et l'hiver la plus courte.

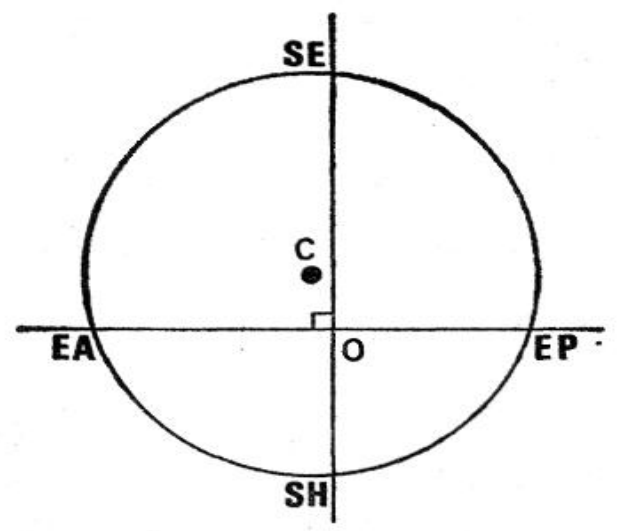

Fig. 2. - Illustration de la théorie solaire d'Hipparque Le centre C est placé

de façon à sauver les longueurs des saisons à notre époque

$\mathrm{EP}=$ équinoxe de printemps, $\mathrm{SE}=$ solstice d'été, $\mathrm{EA}=$ équinoxe d'automne, $\mathrm{SH}=$ solstice d'hiver. 


\section{Mouvement de Mars : deux inégalités}

Dans le cas du Soleil, il n'y a qu'une inégalité - l'inégalité zodiacale. La vitesse angulaire du Soleil varie selon sa position sur le zodiaque.

Dans le cas des planètes, il y a, outre l'inégalité zodiacale, une autre inégalité, ce qui produit le phénomène très frappant du mouvement rétrograde. Or une planète donnée ne rétrograde pas toujours au même point du zodiaque, de sorte que cette inégalité n'est pas une inégalité zodiacale, mais une inégalité d'un autre type. En effet, les rétrogradations d'une planète ne sont pas liées à sa position sur le zodiaque, mais à sa position par rapport au Soleit. C'est ainsi que les planètes supérieures (Mars, Jupiter et Saturne) arrivent toujours au centre de leurs arcs rétrogrades quand elles sont en opposition avec le Soleil; et c'est pourquoi l'inégalité dans le mouvement des planètes qui produit les rétrogradations est appelée l'inégalité par rapport au Soleil.

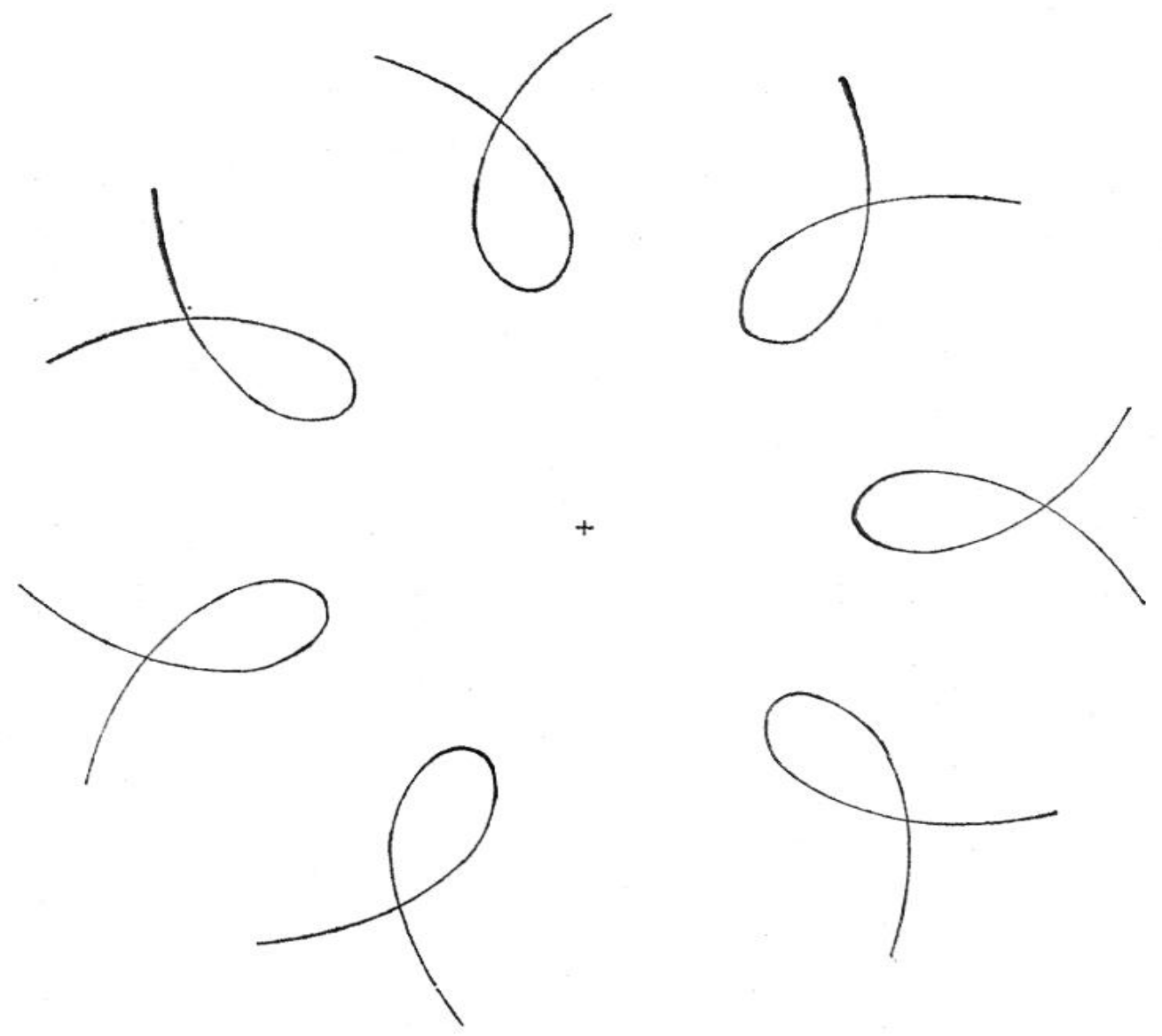

Fig. 3. - Boucles rétrogrades

produites par le modèle d'excentricité zéro de la fig. 4 
L'inégalité zodiacale est appelée, elle, première inégalité. L'origine de cette terminologie se trouve dans la théorie solaire. Le Soleil n'a qu'une inégalité. L'autre inégalité propre aux planètes (celle qui produit les rétrogradations) s'appelle très logiquement deuxième inẻgalité.

Chez les astronomes anciens, c'est l'épicycle qui produit le mouvement rétrograde et qui donc sauve la deuxième inégalité. La révolution de la planète sur l'épicycle produit une série de boucles qui explique, d'une manière générale, les rétrogradations de la planète. Voir la figure 3. Sur cette figure, que l'on trouve fréquemment dans les manuels d'histoire de l'astronomie, les boucles rétrogrades ont la même grandeur et la même forme, et les intervalles entre elles sont uniformes. Cependant, le modèle qui engendre de telles boucles n'est pas le modèle complet de Ptolémée, donné par la figure 1, mais plutôt le modèle simplifié de la figure 4. Dans ce modèle simple, les trois points - point équant, centre du déférent, et Terre - sont confondus. Il n'y a qu'un seul centre. Nous l'appellerons le modèle d'excentricité zéro.

Nous avons construit la figure 3 , qui correspond au modèle d'excentricité zéro, en utilisant des éléments particuliers à Mars. Le rayon de l'épicycle vaut 0,66 fois le rayon du déférent. Le centre de l'épicycle se déplace de $114^{\circ}$ sur le déférent pendant que la planète se déplace de $100^{\circ}$ sur l'épicycle. Entre deux boucles rétrogrades successives, Mars parcourt donc un tour complet de l'écliptique, plus

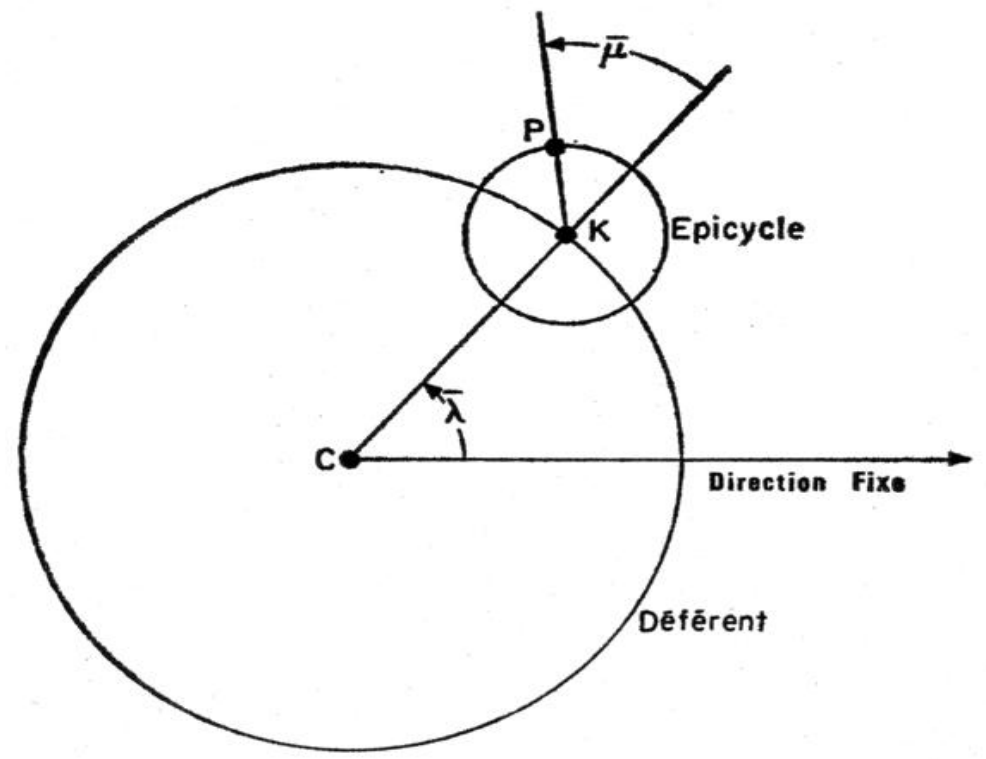

Fig. 4. - Version d'excentricité zéro du modèle de Ptolémée 
un arc de $50^{\circ}$ environ. Le tracé qui correspond au modèle d'excentricité zéro est simple et esthétique. Comme nous l'avons dit, toutes les boucles rétrogrades ont la même grandeur et sont séparées par des intervalles égaux de $50^{\circ}$ : cela veut dire que la planète n'a pas d'inégalité zodiacale, ce qui est très loin de la réalité.

La figure 5 montre les vraies rétrogradations de Mars, sur le fond du ciel, entre les années 1971 et 1984. Le plan de la figure est le plan de l'écliptique. Les longitudes célestes augmentent en sens inverse des aiguilles d'une montre. La Terre se trouve au point $O$. Mars se

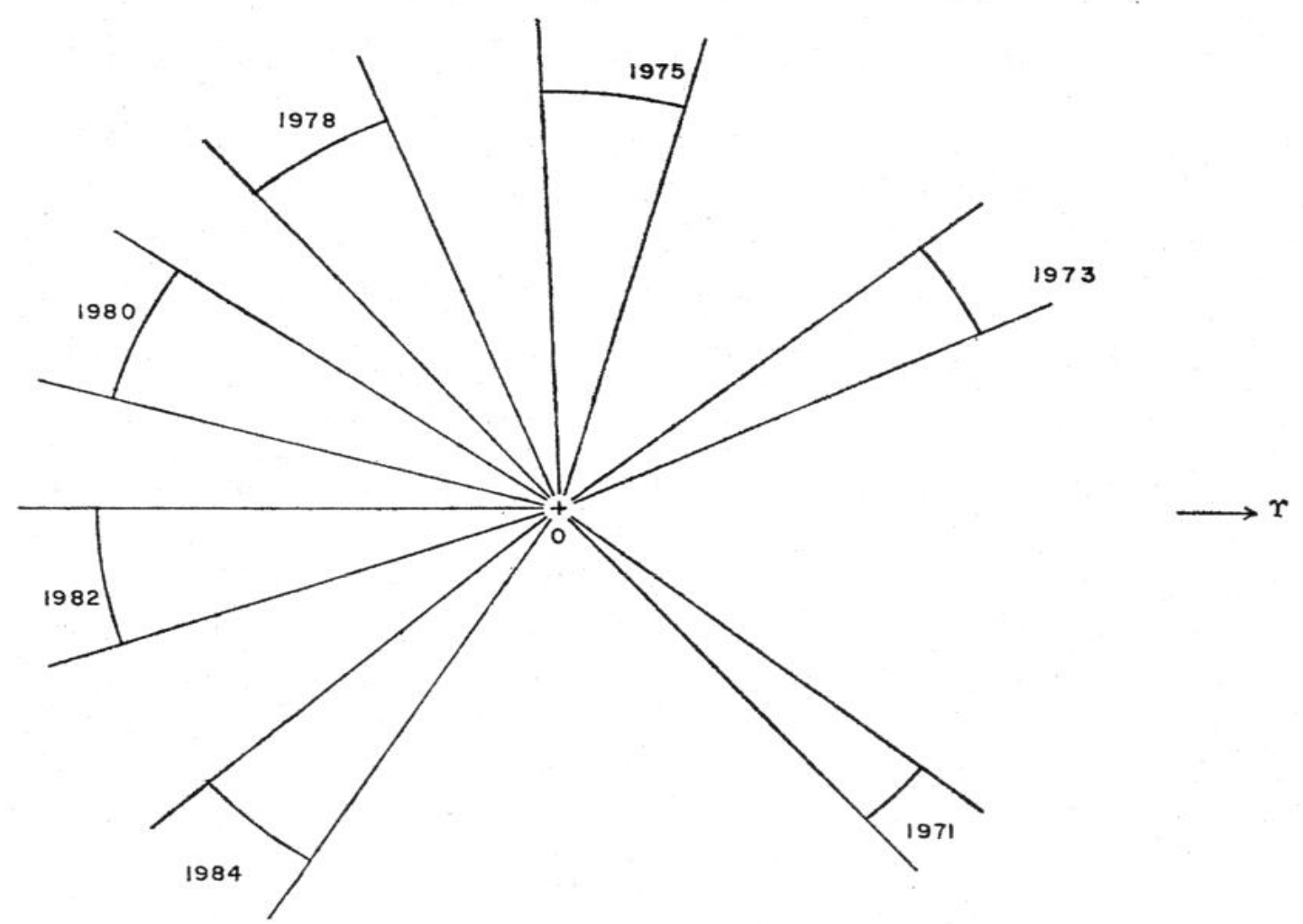

Fig. 5. - Arcs rétrogrades de Mars pour la période 1971-1984 La Terre, d'où l'on fait toutes les observations, se trouve en 0 $\boldsymbol{Y}$ indique la direction de l'équinoxe de printemps

déplace généralement en sens inverse des aiguilles d'une montre, mais de temps en temps la planète rétrograde. L'arc de rétrogradation est de $10^{\circ}$ au minimum (1971) et de $20^{\circ}$ au maximum (1978). Entre deux rétrogradations successives, Mars parcourt complètement autour de l'écliptique, plus un petit arc supplémentaire. Comme cet arc supplémentaire est très variable, les intervalles entre les arcs rétrogrades sont, eux aussi, variables. La distance entre les centres des arcs rétrogrades de 1971 et 1973 est de $75^{\circ}$ (on peut mesurer l'angle directement 
sur la figure). Entre les centres des arcs de 1978 et 1980 , on ne trouve que $34^{\circ}$. C'est une manifestation très frappante de l'inégalité première (ou zodiacale) de Mars.

Le modèle d'excentricité zéro s'accorde-t-il avec le mouvement véritable de la planète? Pour répondre à cette question, nous superposerons le tracé des boucles théoriques (fig. 3) et le tracé des arcs véritables (fig. 5). Il en résulte la figure 6 . La correspondance est à l'évidence très mauvaise. Nous avons pris comme point de départ la boucle de 1971; sa position est, en conséquence, juste. Mais la

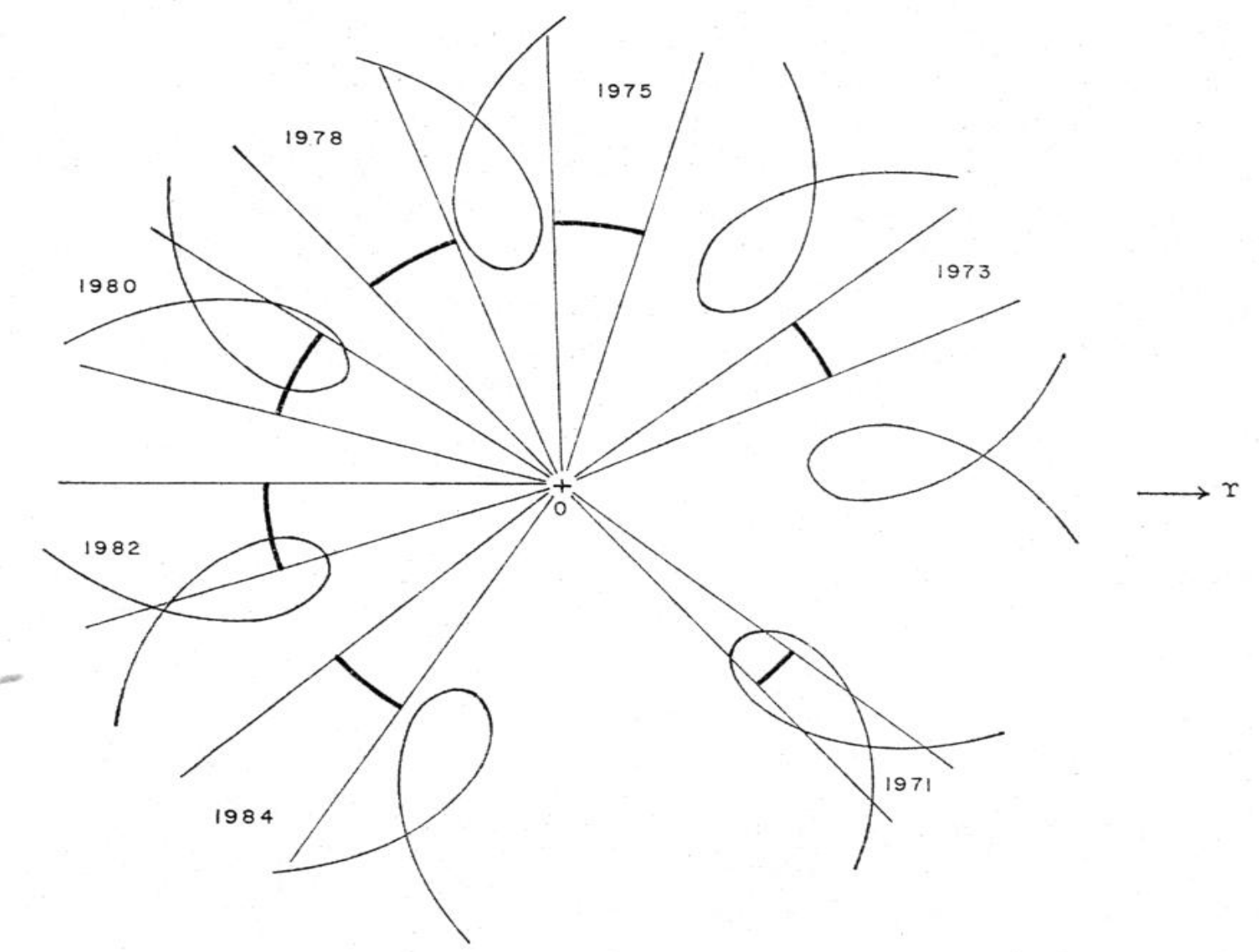

FIG. 6. - Boucles rétrogrades produites par le modèle d'excentricité zéro de Mars superposées aux arcs rétrogrades véritables

(cette figure provient de la superposition des fig. 3 et 5 )

boucle suivante (celle de 1973) occupe une mauvaise position, très en avance sur l'arc réel; et il en va de même pour presque toutes les autres boucles. De plus la largeur des arcs pose également un problème. L'arc réel de 1980 est deux fois plus large que l'arc de 1971. Le modèle d'excentricité zéro ne comporte aucun mécanisme pour produire une telle variation. On peut choisir le rayon de l'épicycle 
afin d'obtenir des boucles qui s'accordent avec la largeur moyenne des arcs rétrogrades observés. Mais il en résulte que dans chaque cas particulier la boucle théorique uniforme sera ou trop large ou trop étroite. Donc, par exemple, la boucle de 1971 est beaucoup trop large et la boucle de 1980 est trop étroite.

NÉCESSITÉ dU SYSTÈME COMPLET DE PTOLÉMÉE DÉMONTRÉE A PARTIR DES APPARENCES

Nous montrerons maintenant comment on aboutit au modèle complet de Ptolémée quand on essaye d'expliquer les inégalités dans les mouvements des planètes que nous venons de voir.

Comme on l'a déjà vu, le modèle simple (d'excentricité zéro) ne peut sauver ni les arcs inégaux, ni les intervalles inégaux entre les arcs. Comment aménager le modèle ? Nous pouvons peut-être emprunter le modèle excentrique à la théorie du Soleil et par suite déplacer le centre du déférent de Mars à une certaine distance de la Terre. Mais dans quelle direction ? Si on regarde attentivement la figure 5 , on remarque une ligne de symétrie très frappante. Cette ligne de symétrie part de l'arc rétrograde de 1971, passe par la Terre $O$ et par l'espace entre les arcs de 1978 et 1980. Il est donc évident que l'on peut déplacer le centre du déférent de Mars le long de cette ligne de symétrie, soit du côté de l'arc de 1971, soit du côté des arcs de 1978 et de 1980.

\section{Un modèle intermédiaire}

Le résultat est un modèle d'une complexité moyenne, un peu plus compliqué que le modèle d'excentricité zéro, mais moins compliqué que le modèle final de Ptolémée.

Ce modèle intermédiaire correspond à la figure 7 . Le centre $K$ de l'épicycle se déplace à une vitesse uniforme sur le déférent; mais le centre $D$ du déférent ne coïncide pas avec la Terre $O$. Il y a deux formes possibles de ce modèle, correspondant aux deux directions possibles du point $D$. Sur la figure $7 \mathrm{~A}$, le centre du déférent se trouve vers l'arc rétrograde de 1971 ; sur la figure $7 \mathrm{~B}$, il se trouve vers la rétrogradation de 1980.

Quels mouvements proviennent du modèle intermédiaire dans chacune de ses versions? La réponse n'est pas difficile. Toutes les boucles rétrogrades auront la même grandeur et la même forme, mais le 


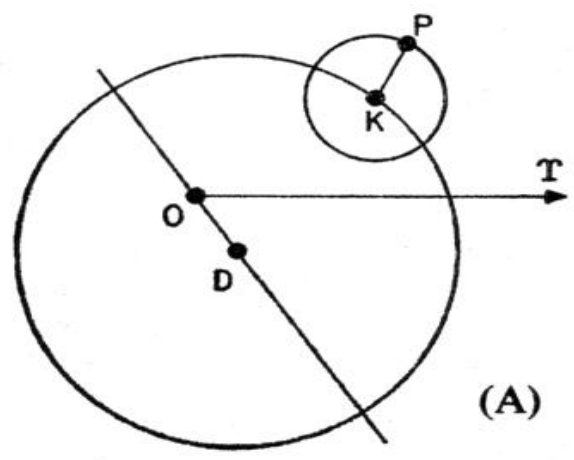

(B)

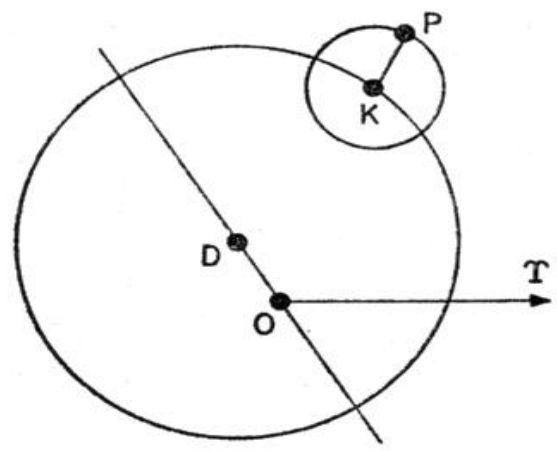

FIg. 7. - Les deux versions du modèle intermédiaire pour Mars. Il n'y a pas de point équant, mais le centre $\mathrm{D}$ du déférent a été déplacé par rapport à la Terre $\mathrm{O}$ dans un sens ou dans l'autre le long de la ligne de symétrie de la fig. 5.

centre de l'ensemble des boucles se trouvera au point $D$ et non plus au point $O$. Nous pouvons comparer la théorie nouvelle avec les données de l'observation en superposant les figures 3 et 5 : il suffit de déplacer le centre de la figure 3 par rapport au centre de la figure 5. Le centre de la figure 3 est $D$, centre du cercle déférent de Mars. Mais le centre de la figure 5 est la Terre $O$, d'où nous observons le ciel.

Essayons d'abord la version du modèle intermédiaire de la figure 7A, sur laquelle le point $D$ se trouve vers l'arc rétrograde de 1971 (voir la fig. 8A). Les largeurs des arcs sont maintenant acceptables. On remarque que les boucles de 1971 et 1980 remplissent presque exactement l'intervalle de la rétrogradation réelle. Donc, en déplaçant le centre du déférent vers la rétrogradation de 1971, nous obtenons un modèle qui produit des boucles de largeurs convenables dans deux régions diamétralement opposées du ciel. Malheureusement, les intervalles entre les arcs sont encore plus inexacts.

Examinons maintenant le mouvement engendré par le modèle intermédiaire de la figure 7B, dans lequel le centre du déférent est cette fois-ci déplacé vers l'arc de rétrogradation de 1980. La figure 8B montre le résultat de la superposition des boucles théoriques et des arcs rétrogrades véritables. Toutes les boucles théoriques se trouvent maintenant sur les arcs rétrogrades correspondants. On constate que la version $B$ du modèle intermédiaire produit les intervalles variables dont nous avons besoin. Qu'en est-il des amplitudes ? En déplaçant le centre du déférent vers la boucle rétrograde de 1980 , nous n'avons pas résolu le problème. La boucle de 1971 est beaucoup trop large, et les boucles de 1978 et 1980 sont beaucoup trop étroites. En effet, le désaccord est plus mauvais qu'autrefois. 


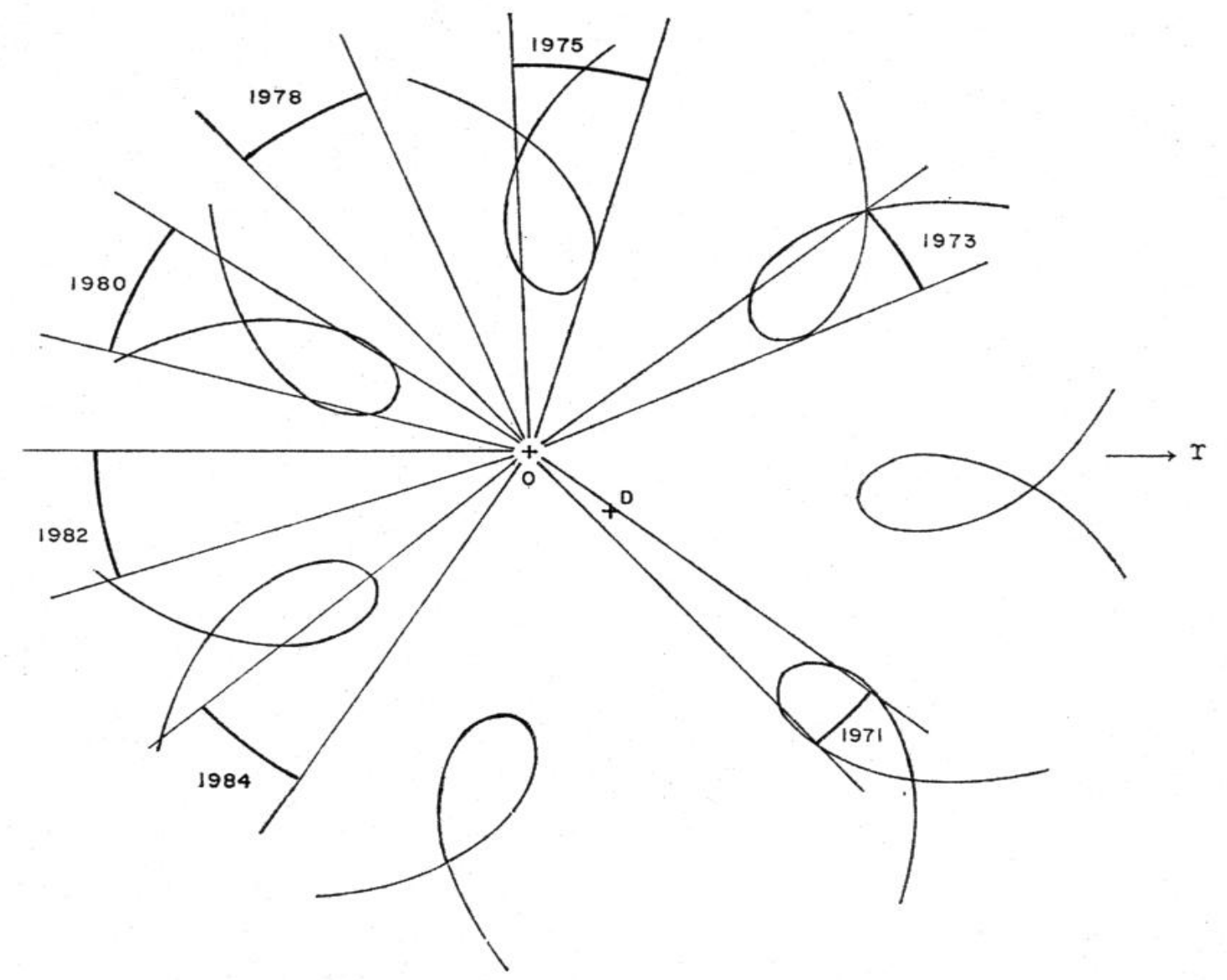

FIG. 8A. - Comparaison du modèle intermédiaire A avec le mouvement réel de Mars

Il semble donc qu'un simple déplacement du centre du déférent ne peut sauver notre modèle. Afin d'obtenir des intervalles variables et exacts entre les rétrogradations, il faut déplacer le centre du déférent dans une certaine direction; mais, pour obtenir des arcs rétrogrades d'amplitudes variables et exacts, il faut déplacer le centre du déférent dans la direction opposée. Il n'y a pas moyen d'obtenir à la fois des intervalles et des arcs exacts par un simple déplacement du centre. On peut illustrer la situation de la façon suivante :

Imaginons une rangée d'arbres. Si nous nous en éloignons, ils paraîtront plus petits et plus proches les uns des autres. Mais, comme on peut le voir sur la figure 5, près de la rétrogradation de 1971, il nous faut des arcs qui sont en même temps petits et éloignés les uns des autres. On ne peut produire cette apparence en déplaçant simplement la position de la Terre par rapport au centre des boucles uniformes de la théorie de l'excentricité zéro. Il nous faut trouver un nouveau mécanisme. 


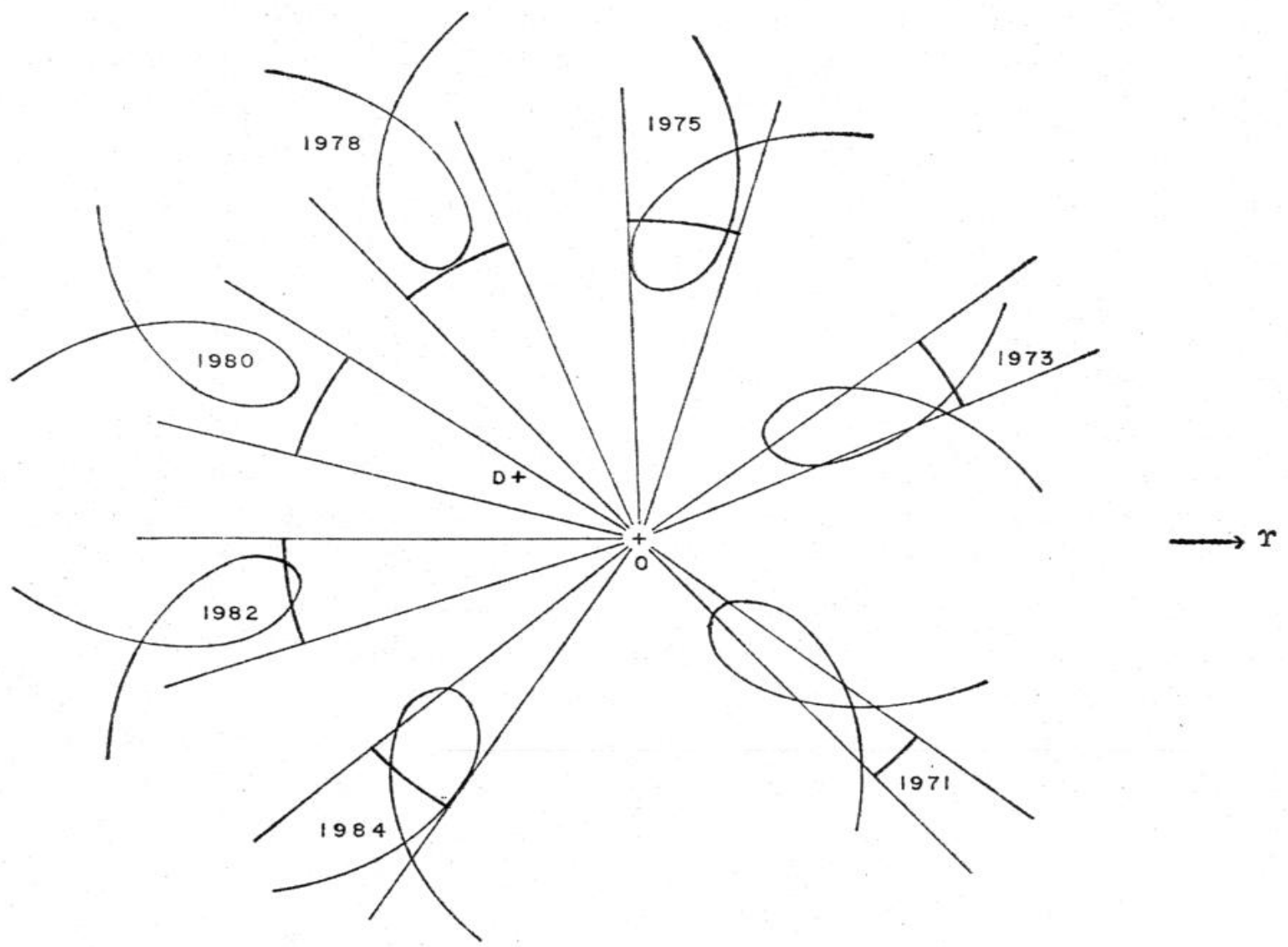

FIG. 8B. - Comparaison du modèle intermédiaire B avec le mouvement réel de Mars

\section{Le modèle final de Ptolémée}

La figure 1 illustre la théorie des longitudes adoptée par Ptolémée pour Vénus, Mars, Jupiter et Saturne. En fin de compte, nous examinerons le mouvement engendré par ce modèle ; mais d'abord, voyons comment le modèle lui-même est suggéré bien directement par un examen des phénomènes des rétrogradations. Nous partons de la version $B$ du modèle intermédiaire (fig. 7B). Le centre du déférent et le centre du mouvement uniforme coïncident au point $D$, qui est légèrement excentré par rapport à la Terre $O$. Autrement dit, le centre $\mathrm{K}$ de l'épicycle se déplace à une vitesse uniforme sur le déférent, et la ligne DK tourne à une vitesse angulaire constante. Sur la figure $8 \mathrm{~B}$, nous avons choisi la position du point $\mathrm{D}$ (centre de l'ensemble des boucles) de manière à obtenir le meilleur accord des boucles théoriques et des arcs observés. Les positions des boucles sont excellentes. Chaque boucle théorique se trouve sur son arc 
rétrograde correspondant. Les boucles, vues du point $D$, sont séparées par des intervalles égaux ( $D$ est le centre du système des boucles). Puisque les rétrogradations véritables coïncident avec les boucles, il en résulte que le point $D$ est vraiment le centre du mouvement uniforme de la planète. En d'autres termes, le point $D$ agit comme un point équant. Les apparences ne laissent pas d'autre choix.

En revanche, le modèle intermédiaire $B$ ne peut produire les amplitudes exactes des arcs rétrogrades. La boucle théorique de 1971 est beaucoup trop grande, et les boucles de 1978 et de 1980 sont beaucoup trop petites. La solution qui s'impose est celle que Ptolémée a adoptée. Il faut séparer le centre du déférent du centre du mouvement à vitesse uniforme. Sur la figure $8 B$, le point $D$ représente l'équant et le centre du déférent. Il faut laisser l'équant en $D$, afin de préserver les intervalles exacts entre les rétrogradations; mais si nous déplaçons le centre du déférent vers $O$, les boucles rétrogrades de 1978 et de 1980 se rapprocheront de la Terre et elles paraîtront, en conséquence, plus grandes. La boucle de 1971 s'éloignera de la Terre et elle paraîtra un peu plus petite. Nous pourrons peut-être obtenir ainsi des largeurs exactes tout en préservant les intervalles exacts que nous avons déjà obtenus. Le modèle final produit par le dédoublement des fonctions du point $D$ réparties entre les deux points $\mathrm{E}$ et $\mathrm{C}$ n'est rien d'autre que le modèle adopté par Ptolémée.

Ce qui provient de ce nouveau modèle, qui sépare le centre du déférent du centre du mouvement uniforme, n'est pas évident ; car les boucles rétrogrades cessent d'avoir une forme et une grandeur uniformes. Maintenant, il faut calculer les mouvements correspondant à ce modèle en utilisant les paramètres propres à Mars. Ces paramètres sont : le rayon de l'épicycle, la valeur de l'excentricité du centre du déférent et la direction de celui-ci par rapport à la Terre, les deux vitesses (de la planète sur l'épicycle et de l'épicycle sur le déférent), et les positions des points $K$ et $P$ à n'importe quel moment donné. Quand nous superposons les boucles rétrogrades qui proviennent du modèle sur les arcs rétrogrades véritables de Mars, nous obtenons la figure 9.

Les positions et les largeurs des rétrogradations sont excellentes. Nous devrions aussi mentionner que le modèle sauve les moments des rétrogradations. Comme on l'a déjà remarqué, les planètes supérieures arrivent toujours au centre de leurs arcs rétrogrades quand elles sont en opposition, ou à peu près, avec le Soleil. La position du Soleil sur le zodiaque est la mesure du temps. Il en résulte que, si 


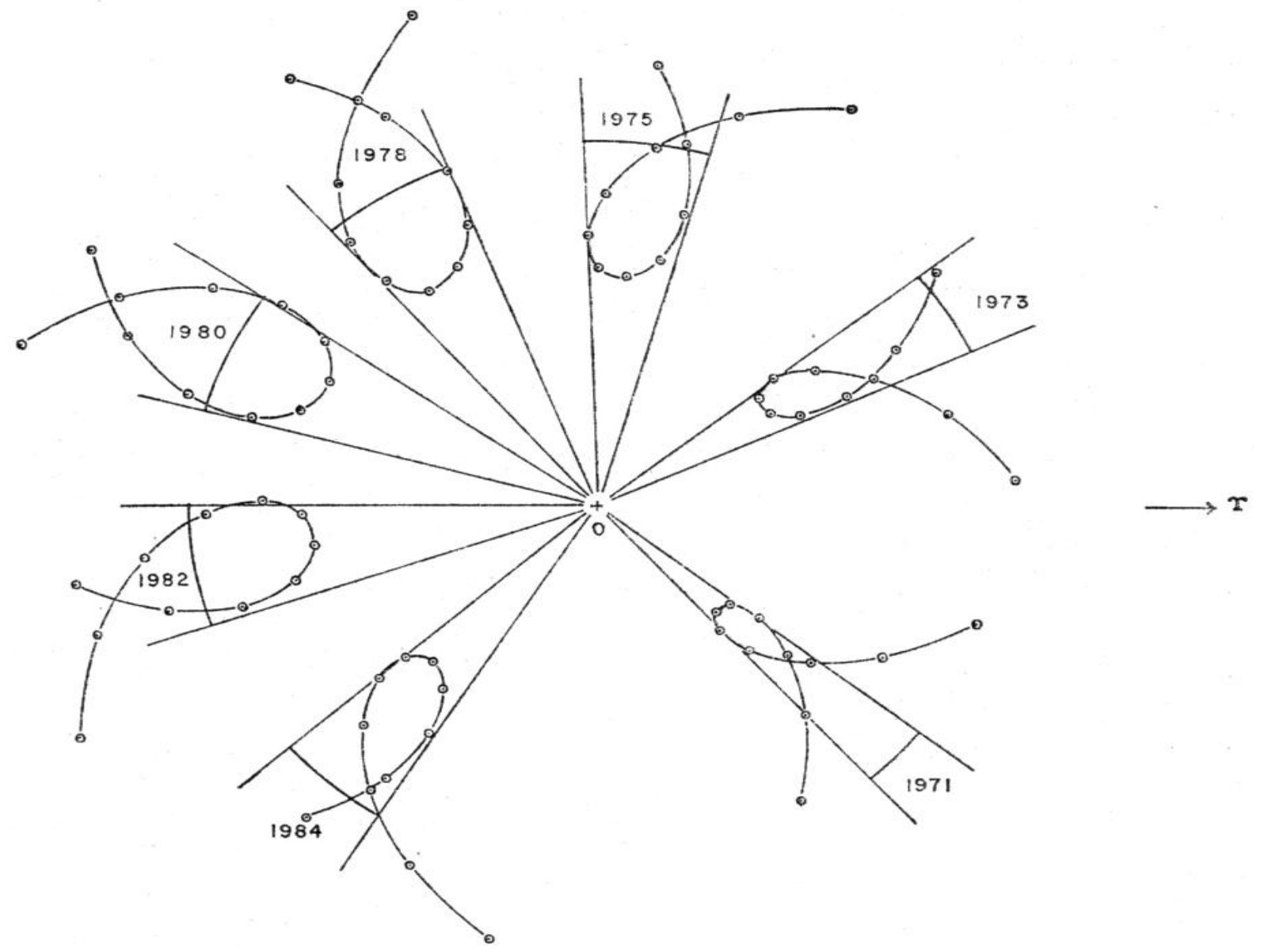

FIG. 9. - Comparaison de la théorie complète de Ptolémée avec les arcs rétrogrades de Mars pour les années 1971-1984 (les paramètres numériques du modèle ne sont pas ceux de Ptolémée, mais des paramètres tirés des données modernes).

les boucles rétrogrades occupent des positions justes sur le zodiaque, les moments des rétrogradations seront en bon accord avec les moments observés. Le modèle final de Ptolémée atteint donc son objectif.

DÉCOUVERTE DE L'ÉQUANT

De l'histoire de la théorie des planètes avant Ptolémée, on ne sait que peu de chose. L'astronomie mathématique dans l'Antiquité comme aujourd'hui était réservée à des spécialistes peu nombreux. Après que Ptolémée eut trouvé la forme finale de la théorie des planètes, les œuvres de ses prédécesseurs perdirent de leur intérêt. Personne ne les lut, personne ne les copia. Presque tout ce que nous savons de 
l'histoire de la théorie planétaire avant Ptolémée dépend des quelques remarques que fait Ptolémée lui-même dans l'Almageste.

Nous essayerons d'esquisser le développement probable de la théorie des planètes jusqu'à la découverte du point équant par Ptolémée. Nous émettrons l'hypothèse que ce développement a suivi à peu près le cheminement que nous venons d'ébaucher. Par là, nous ne voulons pas dire que les astronomes grecs ont dessiné des figures de boucles semblables aux nôtres, mais simplement que c'est le désir de sauver les longueurs des arcs rétrogrades et les intervalles entre eux, dans le cas des planètes supérieures, qui conduisit Ptolémée à son modèle final.

Nos figures rendent visibles d'un seul coup d'œil tous les résultats de centaines de calculs. Nous avons utilisé des observations et des paramètres modernes pour construire ces figures, afin d'éviter la question difficile, et en grande partie hors de propos, de la validité et de la précision des observations anciennes. Pour prévenir des objections possibles il suffit de noter que, au moins dans le cas de Mars, le désaccord entre les rétrogradations réelles et le modèle d'excentricité zéro, ou entre les rétrogradations réelles et n'importe quelle version du modèle intermédiaire, est beaucoup plus grand que l'évaluation la plus prudente des erreurs d'observations anciennes, comme les figures le montrent de manière évidente. Il est donc tout à fait possible que le cheminement proposé soit celui qui a été suivi. Il nous reste à démontrer que ce fut le cheminement probable.

Ptolémée dit très clairement que les astronomes grecs n'avaient aucune théorie satisfaisante avant son époque (2). D'après certaines remarques du XII' livre de l'Almageste, il semble que la théorie de l'épicycle et du déférent ait eu son origine chez Apollonius et d'autres mathématiciens de son école (IIr ${ }^{e}$ siècle av. J.-C.). Selon Ptolémée, ces mathématiciens démontrèrent un théorème permettant le calcul de la longueur de l'arc rétrograde. Voir la figure 10 : $O$ est la Terre et le centre du déférent, $K$ le centre de l'épicycle. Soit $f_{\lambda}$ la vitesse angulaire du point $\mathrm{K}$ sur le déférent ( $f_{\lambda}$ est la vitesse angulaire avec laquelle tourne la ligne $O K$ ). Soit $f_{\mu}$ la vitesse angulaire de la planète sur l'épicycle, par rapport à la ligne tournante OK (si la planète se trouve en F, $f_{\mu}$ est la vitesse avec laquelle l'angle FKO diminue).

(2) Ptolémée, Almageste, IX, 2. Ed. critique de J. L. Heiberg, Claudii Ptolemaei Opera quae exstant omnia, t. I et II, Leipzig, Teubner, 1898-1903; trad. angl. par G. J. Toomer, Ptolemy's Almagest, London, Duckworth, 1984. 


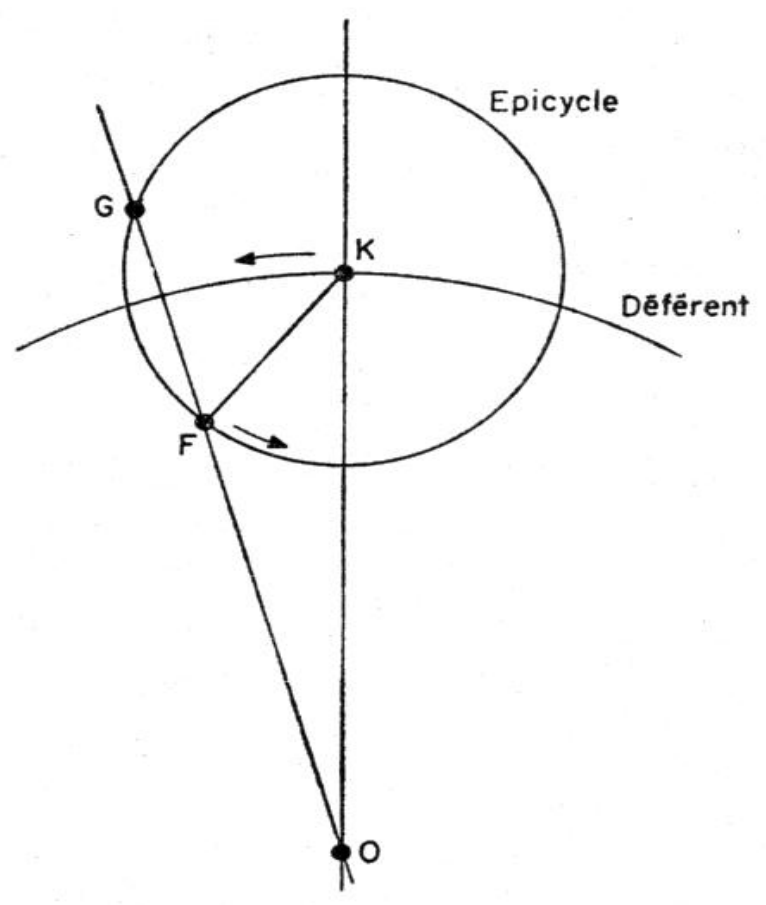

Fig. 10. - Théorème d'Apollonius

Apollonius démontra que la planète, au point $F$, paraît stationnaire, vue du point $O$, quand :

$$
\frac{\mathrm{FG}}{\mathrm{OF}}=\frac{2 f_{\lambda}}{f_{\mu}}
$$

En utilisant ce résultat, on peut calculer la longueur de l'arc rétrograde si l'on connaît les deux vitesses angulaires et le rayon de l'épicycle. Or, les vitesses angulaires sont déterminées par les périodes tropique et synodique de la planète. On peut donc déterminer le rayon de l'épicycle en exigeant du modèle qu'il reproduise des arcs rétrogrades d'une certaine longueur.

Il faut remarquer que le théorème d'Apollonius ne s'applique directement qu'au modèle d'excentricité zéro. Les astronomes qui utilisèrent, les premiers, le modèle du déférent et de l'épicycle ne s'occupèrent à l'évidence que de l'inégalité par rapport au Soleil. Ils n'essayèrent pas d'expliquer l'inégalité zodiacale.

Or, il est presque sûr que ces astronomes connaissaient l'inégalité zodiacale. Comme nous venons de le voir, dans le cas de Mars cette inégalité est très frappante. Il y a une différence de $10^{\circ}$ entre la longueur de l'arc rétrograde le plus long et le plus court. L'intervalle 
entre les centres des arcs successifs varie de $34^{\circ}$ au minimum jusqu'à $75^{\circ}$ au maximum - variation énorme. Il semble donc que les astronomes de l'école d'Apollonius aient négligé à dessein l'inégalité zodiacale et n'aient tenté de donner qu'un modèle planétaire qualitatif et explicatif. L'idée que l'on peut exiger d'un tel modèle qu'il soit quantitatif et prédictif ne dut se former que lentement.

C'est Hipparque qui, le premier, imposa une telle exigence à la théorie planétaire. Malheureusement, toutes les œuvres d'Hipparque ont été perdues, sauf son commentaire sur les Phénomènes d'Aratos. Tout ce que nous savons de ses idées sur la théorie des planètes est tiré de quelques remarques de Ptolémée. D'après Ptolémée, Hipparque apporta une contribution importante aux théories du Soleil et de la Lune, mais il n'essaya pas de donner une théorie des planètes (Almageste, IX, 2). Selon Ptolémée, Hipparque rangea les observations d'une façon plus propre à l'utilisation, et il montra que les phénomènes planétaires ne s'accordaient pas avec les théories des astronomes antérieurs. Hipparque constata que les rétrogradations des planètes ne sont pas uniformes, parce qu'il y a deux inégalités, mais que les mathématiciens avaient donné pourtant leurs démonstrations comme si tous les arcs rétrogrades avaient la même longueur. De cette remarque de Ptolémée, il ressort qu'une partie de la contribution d'Hipparque fut la démonstration que le modèle d'excentricité zéro de ses prédécesseurs était en désaccord avec les mouvements des planètes. Encore peut-on penser que les prédécesseurs immédiats d'Hipparque savaient très bien que les intervalles entre les arcs rétrogrades ne sont pas égaux, sans toutefois en tenir le moindre compte. L'affirmation d'Hipparque, disant que l'on pouvait exiger un modèle planétaire capable de sauver cette inégalité, fut probablement plus importante pour le développement de l'astronomie que son simple constat de l'échec de la théorie existante.

Ptolémée dit aussi qu'Hipparque craignait que les phénomènes ne puissent être sauvés, ni au moyen d'un cercle excentrique, ni au moyen d'un cercle concentrique mais portant un épicycle, ni même au moyen d'une combinaison d'un cercle excentrique avec un épicycle. Un modèle faisant appel à un cercle excentrique et à un épicycle aurait ressemblé aux modèles de la fig. 7. Cela veut dire que, pendant ou avant l'époque d'Hipparque, le modèle intermédiaire fut aussi pris en considération.

Nous pouvons même dire quelle version de ce modèle fut préférée. Pline, au II livre de son Histoire naturelle, donne quelques indica- 
tions sur la théorie des planètes (3). Pline n'était pas astronome : il est à la fois embrouillant et embrouillé ; mais il a écrit quelque soixante-dix ans avant Ptolémée et il connaît des œuvres antérieures à Ptolémée qui ont été perdues. Or Pline remarque que les cercles des planètes n'ont pas le même centre, et il précise que l'apogée de Mars se trouve dans le Lion; l'apogée de Jupiter dans la Vierge; et l'apogée de Saturne dans le Scorpion. Il s'agit donc de la version B : ces apogées sont corrects si on adopte une théorie qui sauve les intervalles entre les rétrogradations, ce qui veut dire que l'on n'avait donc pas essayé de sauver les longueurs des arcs.

Le choix de la version $B$ était du reste le plus raisonnable, car, sauf dans le cas de Mars, la variation dans les longueurs des arcs rétrogrades n'est pas très frappante. Pour la plupart des planètes, il n'y avait donc aucune nécessité urgente de sauver cette variation. Mais les intervalles inégaux sollicitaient l'attention. De plus, il y a une analogie entre la version $B$ du modèle intermédiaire et la théorie solaire. Le centre de l'épicycle de la planète (ou l'épicycle lui-même) tient la place du Soleil. On obtient le mouvement apparent correct de l'épicycle autour du déférent en plaçant l'apogée du déférent dans la région du ciel où ce mouvement est en apparence le plus lent.

Hipparque dut démontrer que, bien que la combinaison des épicycles et des excentriques puisse produire des intervalles corrects, elle ne peut pas produire des arcs rétrogrades de longueurs exactes. Mais, puisqu'il n'a rien proposé pour la remplacer, le modèle intermédiaire resta en vigueur jusqu'à l'époque de Ptolémée (4).

Ce fut donc Ptolémée qui probablement introduisit le point équant. Malheureusement Ptolémée ne dit presque rien de la démarche qu'il a suivie. Il utilise directement le point équant sans explication quant à son origine et comme si sa nécessité s'imposait d'elle-même. Il en est presque toujours ainsi : le mode d'exposition ne retrace presque jamais le cheminement de la découverte.

Il faut cependant relever que, dans son traitement de Vénus,

(3) Histoire naturelle, II, 63-64.

(4) Il y a un vestige du modèle intermédiaire $B$ dans le traitement des planètes supérieures par Ptolémée. Dans le cas du modèle final (avec un point équant), le calcul de l'excentricité à partir de trois oppositions est très difficile. Ptolémée commence donc en supposant que le point équant coïncide avec le centre du déférent, c'est-à-dire qu'il utilise le modèle intermédiaire B. Après avoir obtenu une valeur approximative de l'excentricité par ce calcul, il calcule la correction exigée en passant au modèle final (Almageste $\mathrm{X}, 7 ; \mathrm{XI}, 1 ; \mathrm{XI}, 5$ ). Dans la première étape de ce calcul, on voit que Ptolémée a utilisé la méthode traditionnelle pour déterminer l'excentricité du modèle intermédiaire qu'il a reçue de ses prédécesseurs. 
Ptolémée trouva une méthode pour déterminer directement par le calcul, à partir de certaines observations, la position du point équant (5). Ptolémée montre donc, dans le cas de Vénus, que le centre du mouvement uniforme est séparé du centre du déférent, et que le premier est deux fois plus éloigné de la Terre que le second. On a cru y voir la découverte du point équant (6) : c'est possible mais peu vraisemblable. En effet, contre cette opinion, nous observons que : 1) Avant de commencer son calcul de la position de l'équant de Vénus, Ptolémée devait savoir que l'équant était distinct du centre du déférent, sinon il n'aurait eu aucun motif pour faire le calcul. 2) Ptolémée, se proposant de faire un calcul directement à partir des observations, semble découvrir que le centre du déférent se trouve exactement à mi-chemin entre la Terre et le point équant. Or si la procédure de Ptolémée était fondée sur des données exactes, elle ne mènerait pas à ce résultat précis. Le résultat calculé de Ptolémée n'est donc probablement pas une découverte nouvelle, mais plutôt la confirmation d'une hypothèse qu'il avait déjà conçue. 3) Les dates des observations les plus tardives utilisées pour chaque planète suggèrent, bien que de façon non démonstrative, que Ptolémée a achevé son travail sur les planètes supérieures avant celui sur les planètes intérieures (7). Il faut donc chercher dans une autre direction l'origine du point équant.

Dans le passage de l'Almageste, $\mathrm{X}, 6$, où il commence à traiter des planètes supérieures, Ptolémée affirme sans preuve que pour ces planètes, comme pour Vénus, le centre du déférent se trouve à mi-chemin entre la Terre et le point équant. Puis il ajoute la justification intéressante que l'excentricité calculé à partir de l'anomalie zodiacale est à peu près deux fois plus grande que l'excentricité calculée à partir des longueurs des arcs rétrogrades à l'apogée et au périgée. Il ne donne aucun détail mais il est évident qu'il a expérimenté avec les deux versions du modèle intermédiaire.

En effet, pour au moins une planète supérieure, Ptolémée a calculé l'excentricité qui pouvait sauver l'anomalie zodiacale. Il a trouvé la distance entre la Terre et le centre du déférent (OD sur la fig. 8B) qui donnerait les intervalles exacts entre les arcs rétrogrades. Telle

(5) Almageste, X, 3.

(6) Telle est l'opinion de $O$. Neugebauer, $A$ History of Ancient Mathematical Astronomy, New York, Springer Verlag, 1975, Part I, p. 150-151. Pour d'autres hypothèses sur l'origine du point équant, différente de la nôtre, voir Olaf Pedersen, A survey of the Almagest, Odense, Odense University Press, 1974, p. 277-279, 306-307.

(7) Pedersen, p. 307. 
était la procédure de ses prédécesseurs. Ptolémée a aussi calculé cette même distance dans l'autre version du modèle intermédiaire (OD sur la fig. 8A) qui sauverait les longueurs des arcs rétrogrades. Pour ce dernier calcul, Ptolémée s'est appuyé sur le théorème d'Apollonius. Il a trouvé que les deux résultats n'étaient pas identiques. Comme on peut le voir en comparant les figures $8 \mathrm{~A}$ et $8 \mathrm{~B}$, l'excentricité qui sauve les intervalles est plus grande que l'excentricité qui sauve les longueurs des arcs. Ptolémée dit que la première est deux fois plus grande que la seconde, mais ce n'est en vérité qu'une approximation.

Ptolémée a compris alors qu'il pourrait préserver les intervalles justes en laissant le centre du mouvement uniforme à la distance requise de la Terre, et qu'il pourrait obtenir les rétrogradations exactes en mettant le centre du déférent à la moitié de cette distance.

Que telle ait été la démarche de Ptolémée, c'est ce que l'on ne peut pas prouver. Cependant à titre de confirmation l'on peut mettre en avant un autre fait. Après avoir achevé la construction de la théorie des longitudes des planètes, Ptolémée utilise la théorie nouvelle pour calculer les arcs rétrogrades (Almageste, XII, 1-6). En particulier, il calcule les longueurs des arcs rétrogrades de chaque planète à l'apogée et au périgée, puis il constate que les longueurs des rétrogradations calculées s'accordent avec les longueurs observées (8). Malheureusement, il ne donne pas les valeurs d'observation avec lesquelles on doit comparer les longueurs théoriques. Néanmoins, le contenu du XII e livre montre qu'il était important pour Ptolémée de sauver les longueurs des arcs rétrogrades à l'apogée et au périgée.

Enfin, on peut se demander quelle planète conduisit Ptolémée à sa découverte. Ptolémée dit que deux méthodes indépendantes pour le calcul de l'excentricité donnent deux résultats différents, dont l'un deux fois plus grand que l'autre. Or, dans le cas de Jupiter, les arcs rétrogrades à l'apogée et au périgée sont presque égaux et, en conséquence, le calcul de l'excentricité à partir de ces valeurs n'est pas vraiment possible. Dans le cas de Saturne, la situation n'est guère meilleure. C'est seulement dans le cas de Mars que la différence entre les longueurs des arcs est assez grande pour permettre le calcul. Il est donc probable que Ptolémée a découvert l'équant en luttant avec Mars, la planète qui, quatorze siècles plus tard, deviendra l'adversaire de Kepler.

University of Puget, Tacoma

James Evans.

(8) Almageste, XII, 6 ; trad. B. J. Toomer, p. 581. 